Review

\title{
Examining the Role of Specialized DNA Polymerases in the Development of Temozolomide Resistance in Glioblastoma Multiforme
}

Anthony J. Berdis *

Department of Chemistry, Cleveland State University, 2121 Euclid Avenue, $\mathrm{OH}$ 44115, Cleveland, U.S.; E-Mail: a.berdis@csuohio.edu

* Correspondence: Anthony J. Berdis; E-Mail: a.berdis@csuohio.edu

Academic Editor: Bart Ellenbroek

Special Issue: Novel Approaches to Glioblastoma

OBM Neurobiology

2021, volume 5, issue 2

doi:10.21926/obm.neurobiol.2102096
Received: February 09, 2021

Accepted: May 11, 2021

Published: May 18, 2021

\begin{abstract}
Glioblastoma multiforme (GBM) is an extremely malignant type of primary brain tumor that exhibits a high mortality rate. Current standard therapy involves surgery followed by radiation and treatment with the DNA-alkylating agent, temozolomide (TMZ). While TMZ treatment can extend post-operative survival, most patients develop resistance to TMZ which leads to a significant increase in mortality. At the molecular level, TMZ produces a variety of different DNA lesions including $\mathrm{N}^{7}$-methylguanine, $\mathrm{N}^{3}$-methyladenine, and $\mathrm{O}^{6}$-methylguanine. Although each DNA lesion possesses a unique molecular structure, they all elicit cytostatic and cytotoxic effects against GBM cells. This review article describes the molecular and cellular mechanisms accounting for the anti-cancer activity of TMZ as well as the mechanisms responsible for both inherent and TMZ-induced drug resistance. Special emphasis is placed on understanding the roles that various DNA polymerases play toward the initiation and progression of GBM in addition to mediating resistance to TMZ. This review concludes with discussions on several new approaches that show promise in combating TMZ-resistance, specifically using small molecules to block the replication of DNA lesions catalyzed by various DNA polymerases.
\end{abstract}

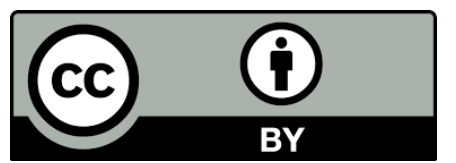

(C) 2021 by the author. This is an open access article distributed under the conditions of the Creative Commons by Attribution License, which permits unrestricted use, distribution, and reproduction in any medium or format, provided the original work is correctly cited. 


\section{Keywords}

Glioblastoma multiforme; DNA damage; DNA polymerization; chemotherapy; nucleoside analogs

\section{Introduction}

Glioblastoma multiforme (GBM) is the most common type of brain cancer and afflicts over 12,000 children and adults each year in the United States [1]. GBM is also the deadliest of all cancers, having 5 -year survival rates of less than $10 \%$ [2]. The current standard of treatment for GBM is referred to as the Stupp protocol published in 2005 [3]. This landmark study demonstrated a survival advantage for concomitant and adjuvant temozolomide (TMZ) treatment with radiotherapy in patients with GBM [3]. This study comprised a total of 573 patients with a median age of 56 years and included only patients with newly diagnosed and histologically confirmed GBM. In addition, the majority of patients (>80\%) had undergone debulking surgery prior to treatment. Patients were randomly assigned to receive a total of $60 \mathrm{~Gy}$ alone (fractionated focal irradiation in daily fractions of $2 \mathrm{~Gy}$ given 5 days per week for 6 weeks) or radiotherapy combined with daily TMZ (75 mg per square meter of body-surface area per day, 7 days per week from the first to the last day of radiotherapy). This was followed by six cycles of adjuvant TMZ (150 to $200 \mathrm{mg} / \mathrm{m}^{2}$ for 5 days during each 28-day cycle). After 28 months, the median overall survival (OS) was 14.6 months in patients receiving a combination of TMZ and radiotherapy which was longer than 12.1 months observed with radiotherapy treatment alone. The two-year OS rate with combining $\mathrm{TMZ}$ with radiotherapy was $26.5 \%$, and this was statistically higher than $10.4 \%$ obtained using radiotherapy alone.

Collectively, these data showed that combining TMZ and radiotherapy provided significant survival benefits for newly diagnosed GBM patients without produced overt toxicities. Since this study was published, TMZ remains the primary FDA-approved drug used to treat GBM [4]. TMZ is an orally administered DNA alkylating agent that can effectively cross the blood-brain barrier [5]. Once inside a tumor, TMZ produces several distinct forms of DNA damage that produce cytostatic and cytotoxic effects. Unfortunately, even with aggressive treatments using IR and TMZ, the median survival time for most GBM patients is less than 16 months [6]. Much of this poor prognosis lies in the development of drug resistance that is caused in part by the pro-mutagenic replication of DNA lesions produced by TMZ. However, a number of new treatments show promise against GBM. For example, carmustine (1,3-bis(2-chloroethyl)-1-nitrosourea (BCNU)) is an anti-cancer agent that also alkylates and cross-links DNA during all phases of the cell cycle [7]. These modifications disrupt DNA replication and transcription to induce cell cycle arrest and/or apoptosis. In addition, carmustine carbamoylates proteins such as DNA repair enzymes, and this modification can impair their activity to increase the cytotoxicity of carmustine. Unlike TMZ, however, carmustine cannot effectively pass through the blood-brain barrier and is thus placed on wafers (Gliadel ${ }^{\circledR}$ ) and used as an intracranial implant to achieve localized chemotherapy $[8,9]$. Another innovative treatment is aldoxorubicin, an analog of the anti-cancer drug doxorubicin (DOX) which is effective against many types of hematological and solid cancers [10]. DOX primarily generates double strand DNA breaks (DSBs), a highly cytotoxic form of DNA damage [10]. Unlike TMZ, DOX is unable to effectively penetrate the blood-brain barrier and is thus not used to treat GBM. However, aldodoxorubicin is a prodrug of 
DOX that contains a $\mathrm{pH}$ sensitive linker that allows for transport across the blood-brain barrier and preferential release of the drug within GBM cells [11]. In fact, results from a recent phase II clinical trial in relapsed GBM patients showed that aldoxorubicin penetrates the blood-brain barrier and is associated with objective tumor responses via MRI imaging and prolonged survival [12]. Finally, the anti-angiogenic agent bevacizumab (Avastin ${ }^{\circledR}$ ) has received attention as second line therapy against GBM. While bevacizumab does not improve overall survival, it does improve the management of symptoms and overall quality of life for GBM patients [13, 14].

It is beyond the scope of this review to critically evaluate all of these therapeutic approaches against GBM. As such, this review focuses on the molecular and cellular mechanisms accounting for the anti-cancer activity of $\mathrm{TMZ}$, mechanisms accounting for $\mathrm{TMZ}$ resistance, and approaches being developed to combat drug resistance. Special emphasis is placed on discussing new approaches to block the replication of DNA lesions that persist after exposure to TMZ.

\section{Results}

\subsection{Molecular and Cellular Mechanisms of Temozolomide}

The chemical mechanism responsible for the ability of TMZ to produce different forms of DNA damage is illustrated in Figure 1. The key step in this reaction is the ability of TMZ to undergo spontaneous hydrolysis at physiological $\mathrm{pH}(\sim 7.4)$ to form a methyldiazonium ion. The formation of this strong electrophile enables an $\mathrm{S}_{\mathrm{N}} 1$-type alkylation reaction in which the methyldiazonium ion effectively reacts with nucleophilic functional groups (oxygen and nitrogen) present on single-strand and duplex DNA [15]. Reactions on duplex DNA predominantly occur primarily at the N7 position of guanine (70\%), the N3 position of adenine (10\%) and the 06 position guanine (7\%) [16]. Additional sites for alkylation include the N1 of adenine and the N3 of cytosine. However, these reactions occur more frequently in single-stranded DNA that typically forms at DNA replication forks during DNA synthesis and at sites of active gene transcription [17].

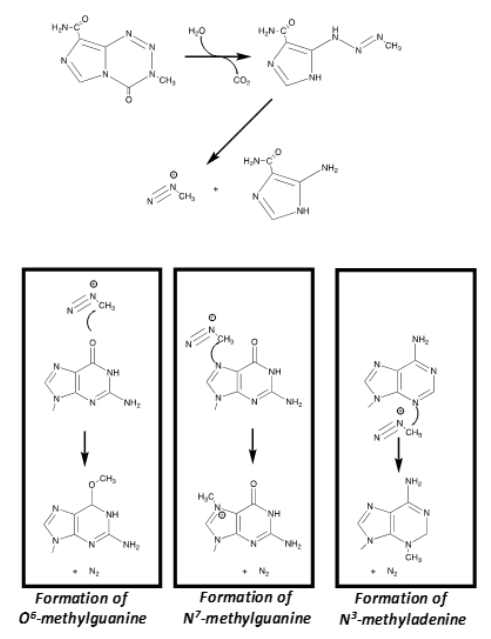

Figure 1 Chemical mechanism for the activation of temozolomide (TMZ) to produce different DNA lesions. TMZ undergoes spontaneous hydrolysis to form a methyldiazonium ion which reacts with nucleophilic functional groups present on singleand double-stranded DNA. Primary sites for modification are the N7 position of guanine, the N3 position of adenine, and the $\mathrm{O} 6$ position of guanine. 
The most commonly formed DNA lesions, $\mathrm{N}^{7}$-methylguanine, $\mathrm{N}^{3}$-methyladenine, and $\mathrm{O}^{6}$ methylguanine, all produce cytostatic and cytotoxic effects. However, since each DNA lesion is structurally unique, the mechanisms accounting for their anti-cancer effects differ at the cellular level. As a consequence, the underlying mechanisms for resistance are different, and these differences have significant ramifications on therapeutic response. DNA damage caused by TMZ induces cellular senescence which is characterized by cell-cycle arrest at the G2/M phase [18]. Recently, Aasland et al. showed that TMZ-induced senescence is initiated by damage recognition through the MRN complex followed by activation of ATR/CHK1 kinases and degradation of CDC25C [19]. However, TMZ-induced senescence also depends upon functional p53 in addition to sustained p21induction. While the NF-KB pathway is also required for TMZ-induced senescence, neither p14 nor p16 (which are targets of p53) do not appear to play essential roles. In addition, TMZ treatment represses expression of several MMR pathway proteins including MSH2, MSH6, and EXO1. Surprisingly, the homologous recombination protein RAD51 is also downregulated. Both effects appear to be dependent upon p53 activity since their repression is not observed in p53-deficient cells.

Although the focus of this review is on drug resistance caused by the replication of lesions produced by TMZ, it is necessary to first understand how these DNA adducts are repaired. Provided below is a brief description of the major DNA repair pathways involved in correcting the three most common lesions formed by TMZ. The cytotoxic effects of a methylated nucleobase are typically caused by the ability of the formed adduct to transiently disrupt the continuity of DNA replication or to block this process completely [10]. For example, methyl adducts such as N3 adenine occur at positions on a nucleobase that directly inhibit DNA synthesis catalyzed by a DNA polymerase. In these cases, methylation at this and other key nucleobase positions disrupt essential contacts between the nucleobase and critical active site amino acid residues to block DNA synthesis [20, 21]. This inhibition causes the formation of blocked replication forks that are unstable and ultimately collapse to produce double-strand breaks (DSBs) which are highly cytotoxic. In contrast, methylation at other nucleobase positions can perturb interactions associated with proper Watson-Crick basepairing interactions. For example, methylation at the $\mathrm{O}^{6}$ position of guanine produces $\mathrm{O}^{6}$ methylguanine that does not permanently block replication fork progression but rather allows for the incorporation of either cytosine or thymine. Neither nucleotide can correctly base-pair with the adduct. As such, the resulting mis-pair is excised and repaired by the mismatch repair (MMR) pathway [22]. As described in more detail later, the MMR pathway works to first excise newly synthesized DNA to produce a long single-strand gap. The gap is then filled in by repair DNA synthesis. However, since the DNA lesion originally present in the template stand is not excised, gap-filling DNA synthesis simply generates another mis-pair. This produces a futile cycle caused by repetition in mis-pair formation, excision, and reformation of a mis-pair. Eventually, the resulting single-strand gap that forms during this futile cycle generates in a DSB during the next S-phase to induce cell death [23].

The most frequently formed DNA lesion is $\mathrm{N}^{7}$-methylguanine [24]. At face value, this lesion would appear to be harmless since the modification occurs at a position in the major groove of DNA which should not directly influence DNA polymerase activity. However, methylation at this and the N3 position of guanine as well as the N7 position of adenine accelerates the rate of non-enzymatic hydrolysis of the glycosylic bond between the nucleobase and deoxyribose [25, 26]. This 
depurination reaction forms a new distinct lesion termed an abasic site which functions as a strong block to DNA synthesis catalyzed by most DNA polymerases [27-29].

\subsection{Repair Pathways for Alkylated DNA}

There are several distinct DNA repair pathways responsible for correcting DNA lesions produced by mono-functional alkylating agents such as TMZ. These include direct repair of $\mathrm{O}^{6}$-methylguanine by the enzyme $\mathrm{O}^{6}$-methylguanine methyltransferase (MGMT), the mismatch DNA repair (MMR) pathway, and the base excision repair (BER) pathway. In normal cells, these pathways are essential for protecting the nuclear genome as they correct methylated and other types of DNA lesions that arise from endogenous and exogenous sources. However, their activity has long been demonstrated to have deleterious effects on patient responses to $T M Z$ by promoting drug resistance [30-32]. In addition, defects in the MMR and BER pathway can produce equally devastating effects by allowing DNA lesions caused by TMZ to persist. This allows the unrepaired DNA lesions to be misreplicated, and this process can drive drug resistance and/or mutagenesis. The MGMT, MMR, and BER pathways have been extensively reviewed elsewhere [33-38]. However, provided below are brief descriptions of each pathway to emphasize their roles in generating TMZ-resistance, particularly with the inappropriate replication of unrepaired DNA lesions.

\subsection{Direct Repair by $0^{6}-$ Methylguanine-DNA Methyltransferase}

$\mathrm{O}^{6}$-methylguanine DNA methyltransferase (MGMT) directly corrects damaged guanine nucleobases by transferring the methyl group from the $\mathrm{O}^{6}$ position of guanine to an active site cysteine residue [39]. MGMT is considered a "suicide" DNA repair enzyme since methylation of the active site cysteine inactivates the enzyme. This limits MGMT to a single-round of DNA repair as opposed to catalyzing multiple turnovers of nucleobase correction [40]. Despite this limitation, MGMT activity is important for maintaining genomic integrity by preventing mutagenesis and possible tumorigenesis caused by DNA alkylation. While the importance of MGMT activity in a normal cell is intuitively obvious, the loss of MGMT activity can have surprisingly beneficial outcomes for cancer patients being treated with TMZ. For example, molecules such as $\mathrm{O}^{6}$ benzylguanine have been used to inhibit MGMT activity, and this inhibition can sensitize cancer cells to the cell killing effects of DNA alkylating agents [41-43]. In addition, MGMT expression is an important feature in therapeutic responses to TMZ. Several studies have shown that the loss of MGMT expression is not due to gene deletion, mutation, rearrangement or unstable RNA, but due to methylation of CpG island of MGMT promoter [44-46]. In fact, epigenetic silencing of the MGMT promoter methylation is associated with longer survival in GBM patients receiving TMZ as part of their treatment [47]. Finally, the methylation status of the MGMT promoter is proposed to be an accurate biomarker in predicting the prognosis of GBM patients [48-50].

\subsection{DNA Mismatch Repair}

The mismatch repair (MMR) pathway is primarily involved in correcting replication errors that have escaped the proofreading activity of high-fidelity DNA polymerases during chromosomal replication [51]. Mismatches fall into two categories, the first being base-base mis-pairs such as $T$ paired opposite $\mathrm{G}$ while the second are insertion and deletion mismatches caused by strand slippage 
at repetitive DNA sequences. As indicated earlier, MMR plays an important role in processing mispairs such as $C$ and $T$ paired opposite $O^{6}$-methylgaunine caused by $T M Z$ exposure. In humans, the MMR pathway features two families of MMR proteins that are heterodimeric homologs of bacterial MutS (MSH) or MutL (MLH). Both proteins have been extensively studied at the biochemical and cellular levels [52-54]. The MMR pathway is generally divided into three distinct phases that include mismatch recognition, excision, and re-synthesis of excised DNA. Each phase is catalyzed by a confederation of proteins, and their activities are tightly regulated to ensure complete and accurate repair of damaged DNA.

The first stage of MMR is mismatch recognition by MutS $\alpha$ or MutS $\beta$. The choice of initiator is dictated by the type of DNA lesion. MutS $\alpha$ initiates the repair of base-base mispairs and small deletions of 1 to 2 nucleotides. In contrast, MutS $\beta$ is primarily responsible for initiating the repair of DNA segments containing larger deletions (1 to15 nucleotides). Binding to a mismatch induces an exchange of ADP for ATP in both MutS $\alpha$ and MutS $\beta$, and this exchange converts both proteins into sliding clamps that allows the proteins to perform a two-dimensional search along DNA to identify other mismatches and to interact with MutL $\alpha$.

The second phase is DNA excision in which the newly synthesized DNA strand containing the error is excised while leaving the templating strand unaltered. Although the mechanism for strand recognition is not completely understood in eukaryotes, recent results suggest that PCNA plays an important role in regulating this process [55]. In vitro and in vivo evidence demonstrate that PCNA can interact with MutS and MutL in addition to other proteins such as EXO1 as well as Pol $\delta$, and Pol $\varepsilon$ which are involved in re-synthesizing excised DNA [56-58]. In vitro MMR reactions require a preexisting nick or single-strand gap in the heteroduplex DNA substrate that can reside on the $5^{\prime}$ - or $3^{\prime}$ - side of the mismatch. In 5'-MMR reactions, excision is catalyzed by EXO1, a 5' to 3' exonuclease that is activated by MutS $\alpha$ in a mismatch-dependent manner. Excision in the case of $3^{\prime}-\mathrm{MMR}$ (a break or gap located on the $3^{\prime}$ side of the mismatch) is less well understood since EXO1 is currently the only exonuclease that appears to be involved in MMR.

The third and final phase of MMR involves DNA gap filling by a high-fidelity polymerase followed by ligation catalyzed by DNA ligase I. These activities collectively generate a corrected and intact DNA duplex. During this process, PCNA is loaded at a $3^{\prime}$ terminus by RFC, and replicative DNA polymerases, pols $\delta$ or pol $\varepsilon$, replicate the RPA-coated gapped DNA. DNA ligase I generates the final phosphodiester bond to fully repair the DNA.

As expected, proper MMR function can be compromised by mutation or epigenetic silencing to generate a hypermutator phenotype, and this can produce devastating pathological effects by altering genomic fidelity and integrity. For example, dysfunctional MMR activity Is responsible for increased susceptibility for inherited cancers such as Lynch syndrome [59].

\subsection{Base Excision Repair Pathway}

The most abundant TMZ-induced adducts, 7-meG, 3-meA, and abasic sites, are repaired by the short patch base excision repair (BER) pathway. The BER pathway primarily functions to repair endogenous forms of DNA damage caused by reactive oxygen species generated during oxidative metabolism [60]. Spontaneously generated forms of damaged DNA include oxidized bases (8oxoguanine), alkylated bases ( $\mathrm{N}^{3}$-methyladenine), abasic sites, and single-strand breaks (SSBs). In a normal cell, it is estimated that as many as 50,000 BER-repaired lesions form per cell during the 
course of a single day [61]. However, treatment with chemotherapeutic agents such as TMZ put a remarkable strain on BER since the number of these lesions increases by several orders of magnitude.

Repair of $\mathrm{N}^{3}$-methyladenine and $\mathrm{N}^{7}$-methylguanine is initiated by recognition of the lesions by alkylpurine-DNA-N-glycosylase (AAG). AGG cleaves the glycosylic bond between the damaged base and deoxyribose to produce an abasic site as the key intermediate in the repair process. After the hydrolysis reaction, AAG remains bound to the abasic site and recruits the endonuclease, Ape1, to the site of damage. Ape1 cleaves the DNA phosphodiester backbone to produce a SSB containing a $3^{\prime}-\mathrm{OH}$ and a $5^{\prime}$ deoxyribose phosphate (5'-dRP) termini [62]. Ape1 is then replaced by DNA polymerase $\beta$, a repair DNA polymerase that possesses $5^{\prime}$ lyase activity that can excise the $5^{\prime}$-dRP to produce a single nucleotide gap. The formed gap is subsequently filled in by DNA polymerase $\beta$, and DNA ligase seals the nick to finalize the repair process.

\subsection{Consequences of Ineffectual DNA Repair}

The most common mechanisms associated with TMZ resistance involve alterations in DNA repair pathways such as MGMT [63] and MMR [64]. As expected, the lack of efficient DNA repair makes cancer cells much more susceptible to mutagenesis which can produce more malignant cancers which possess additional mechanisms of drug-resistance. Indeed, a landmark study by Johnson et al. showed that genomic DNA isolated from recurrent GBM tumors initially treated with TMZ were highly mutated [65] Their results showed that TMZ-treated tumors contained $\sim 30$ to 90 mutations per megabase compared to initial tumors which had significantly lower mutation frequencies $(<4$ mutations per $\mathrm{Mb}$ ). Furthermore, recurrent GBM tumors were drug resistant, and this coincided with the accumulation of acquired somatic mutations in genes associated with MMR, retinoblastoma, and mammalian target of rapamycin (Akt-mTOR) pathways [65]. These results strongly implicate a mechanism in which DNA lesions formed by TMZ are inappropriately replicated due to ineffectual DNA repair.

Unrepaired DNA lesions can be misreplicated by various DNA polymerases in a biological process known as translesion DNA synthesis (TLS). First identified in bacterial cells, the ability to replicate damaged DNA via TLS activity was considered a "necessary evil" by allowing cells to tolerate unrepaired DNA lesions in order to survive [66]. It is now established that TLS is a highly conserved mechanism that occurs in both prokaryotes and eukaryotes [67]. In humans, however, TLS activity is often considered a "double-edged sword" since it can produce both beneficial and adverse effects on genomic fidelity and cellular homeostasis. One example of the beneficial effects of TLS activity comes from the disease known as xeroderma pigmentosum variant (XP-V) which occurs in individuals possessing mutations in a specialized DNA polymerase denoted as polymerase eta (pol п) [68]. This polymerase is unique as it can bypass unrepaired thymine dimers with high efficiency while maintaining genomic fidelity [69]. Individuals lacking pol $\eta$ activity display an increased incidence of squamous cell skin carcinomas in response of UV-exposure and this highlights an important role for replicating damaged DNA [70]. However, unregulated TLS activity can have equally devastating effects as this activity is associated with generating somatic mutations that drive drug resistance to chemotherapeutic agents. As discussed in more detail below, there are several unique challenges toward a complete understanding of how TLS activity drives drug resistance. For example, there are a number of different DNA polymerases that can replicate lesions generated by 
anti-cancer agents such as TMZ. Thus, it is difficult to unambiguously determine which specific DNA polymerase(s) is(are) responsible for generating resistance. In this case, the inherent redundancy in DNA polymerase utilization during TLS represents another set of challenges to develop prognostic biomarkers that accurately correlate TLS activity with TMZ-resistance. In addition, the redundancy may pose challenges toward developing therapeutic agents that block TLS activity in response to chemotherapy.

In humans, TLS activity is believed to be primarily catalyzed by a family of DNA polymerases that include polymerase eta ( $\mathrm{pol} \eta)$, polymerase iota (pol $\mathrm{l}$ ), polymerase theta (pol $\theta$ ), polymerase zeta (pol そ), polymerase kappa (pol k), and the Rev1 protein. These unique polymerases are coined "specialized" due to their ability to replicate a wide variety of structurally distinct DNA lesions with high efficiency and remarkable fidelity. For example, pol $\eta$ displays an usually high error rate of making 1 mistake every 100 opportunities when replicating undamaged DNA [71]. However, pol $\eta$ is highly proficient at replicating naturally occurring DNA lesions such as thymine dimers and abasic sites as well as lesions including $\mathrm{O}^{6}$-methylguanine and cisplatinated DNA that are produced during treatment with anti-cancer drugs [72-74].

The unique activity displayed by specialized DNA polymerases directly contrasts that inherent to "high-fidelity" DNA polymerases such as polymerase alpha (pol $\alpha$ ), polymerase delta (pol $\delta$ ), polymerase epsilon (pol $\varepsilon$ ), telomerase, and polymerase gamma (pol $\gamma$ ). Since these polymerases are intimately involved in chromosomal and mitochondrial DNA synthesis, they display optimal activity on undamaged DNA and low activity with damaged DNA. However, there are reported instances in which high-fidelity polymerases can efficiently replicate small, miscoding DNA lesions such as $\mathrm{O}^{6}$-methylguanine and 8-oxo-guanine [75-77].

Finally, there is a third class of DNA polymerases that primarily function in DNA repair pathways. These include polymerase beta (pol $\beta$ ) which is responsible for processing damaged DNA repaired by BER. In addition, polymerase lambda ( $\mathrm{pol} \lambda$ ) and polymerase mu ( $\mathrm{pol} \mu$ ) play important roles in properly repairing DSBs formed during homologous recombination and non-homologous endjoining [78]. Finally, the fascinating polymerase known as terminal deoxynucleotidyl transferase (TdT) is important for generating immunological diversity by randomly incorporating nucleotides into single strand DNA during $V(D) J$ recombination [79]. Each of these polymerases display variable fidelity depending upon whether they replicate undamaged or damaged DNA.

Aberrant TLS activity has been extensively demonstrated to play a role in tumorigenesis by promoting genetic mutations in addition to driving drug resistance [80-86]. For example, pol $\zeta$ has been shown to be overexpressed in several types of cancers including melanoma [81] and esophageal squamous cell carcinomas [82]. Furthermore, higher levels of pol $\zeta$ correlate with increased resistance to chemotherapeutic agents such as cisplatin which causes intrastand crosslinks $[83,84]$. Likewise, pol $\eta$ overexpression has been shown to be a common factor associated with producing drug resistance to cisplatin in several types of cancers including breast [85] and head and neck squamous cell carcinomas [86].

TLS activity is also associated with tumorigenesis in gliomas and drug-resistance to TMZ [87-89]. In contrast to cancers such as breast and ovarian cancers which show a reliance on the activities of pol $\eta$ and pol $\zeta$ for tumorigenesis and drug resistance, GBM cells appear to rely on the activities of pol $\mathrm{\kappa}$ and pol . For instance, a recent study by Wang et al. examined 40 primary glioma samples and 10 normal brain samples to determine if increased expression of various specialized DNA polymerases contributes to cancer initiation and progression [87]. In this study, expression levels of 
pol $\mathrm{k}$, pol $\mathrm{l}$, and pol $\eta$ were examined using quantitative RT PCR and Western blot analysis. Results using glioma samples showed no significant expression of pol $\eta$. However, overexpression of pol $\iota$ was observed in $\sim 28 \%$ of samples while pol k showed an even higher frequency of overexpression ( $58 \%)$ of samples. The prognostic significance of these higher polymerase levels was confirmed using a population-based tissue microarray derived from a cohort of 104 glioma patients. Immunohistochemical studies showed positive pol $\mathrm{s}$ staining in $\sim 32 \%$ of these glioma specimens while $69 \%$ were positive for pol $\mathrm{k}$ staining. Furthermore, pol $\mathrm{k}$ - and pol t-positive staining correlated with reduced survival within this set of glioma patients. Collectively, these findings highlight how deregulated expression of pol $\mathrm{k}$ and pol $\mathrm{i}$ may stimulate tumorigenesis and provide a poor prognosis for glioma patients.

Following these observations, the same group investigated if overexpression of pol $\mathrm{k}$ also plays a role in generating TMZ-resistance [88]. Their results show that pol $\mathrm{k}$ overexpression confers resistance to the drug in GBM cells that display initial sensitivity to TMZ. Likewise, knocking down pol $\mathrm{k}$ expression in GBM cells resulted in TMZ-sensitivity. The basis for these effects is complex as pol $\mathrm{k}$ depletion caused a cascade of cellular events associated with the maintenance of genomic integrity. These include defective homologous recombination-mediated repair and restart of stalled replication forks which led to the inhibition of cell-cycle re-entry and progression.

While specialized DNA polymerases are considered prime suspects in tumorigenesis and drug resistance, they are not the only culprits. In fact, recent evidence from Campbell et al. suggests that high-fidelity DNA polymerases, pol $\varepsilon$ and pol $\delta$ that are involved in leading and lagging strand DNA synthesis, respectively, may play significant roles in tumorigenesis in cancers including gliomas [90]. This supposition is based on an assessment of mutation burden obtained through sequencing analysis of $>81,000$ tumors from pediatric and adult patients [90]. Their findings show that a large number of pediatric cancers possess defects in MMR pathway genes POLE and POLD1. In addition, ultra-hypermutated pediatric tumors, defined as those possessing greater than 100 mutations per megabase, were all replication repair deficient. These tumors include colorectal cancers, leukemia/lymphomas, and malignant gliomas. In these cases, the authors conclude that while defects in MMR alone can initiate tumorigenesis by increasing mutagenesis, it is the combination of defective MMR coupled with mutations in pol $\delta$ and pol $\varepsilon$ that causes the hyper-mutated state observed in these cancers. Surprisingly, mutations identified in pol $\delta$ and pol $\varepsilon$ do not exclusively occur in the exonuclease-proofreading domain that corrects replication-induced errors. Instead, a wide spectrum of mutations were identified that in regions spanning the polymerization domain, the exonuclease domain, and connection domains. Further studies are needed to correlate specific polymerase mutations with hyper- and ultra-hypermutated genotypes. However, it is clear that mutations in these replicative polymerases alter their intrinsic fidelity and contribute to cancer initiation and drug-resistance.

\subsection{Inhibiting TLS Activity as a New Therapeutic Strategy}

Cancers such as GBM typically possess defects in one or more DNA repair pathways. These defects give a cancer cell only one option to survive the effects of TMZ - it must replicate damaged DNA inflicted by this agent. This leads to the hypothesis that inhibiting the misreplication of DNA lesions that escape DNA repair could be a new therapeutic strategy to improve patient outcomes, especially with respect to TMZ-resistance [91] (Figure 2). 


\section{Biological Problem with Chemotherapy}

Anti-cancer agents create DNA lesions that can be misreplicated

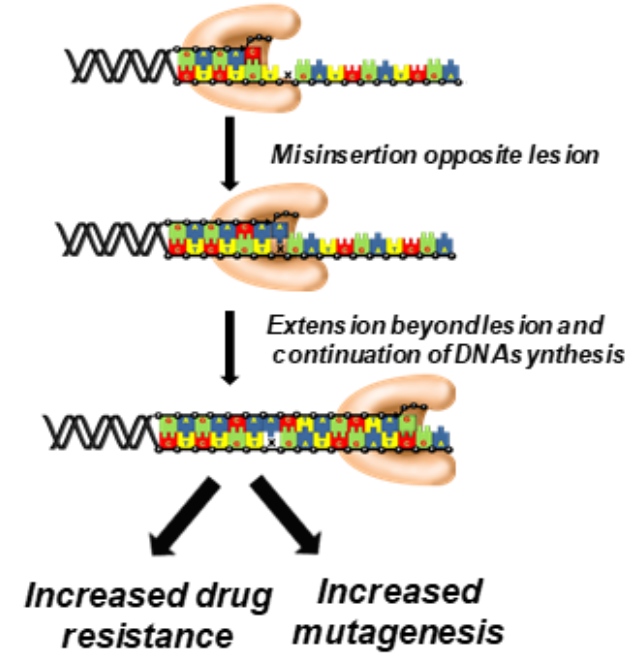

\section{Therapeutic Solution}

Develop non-natural nucleotides that inhibit the replication of damaged DNA

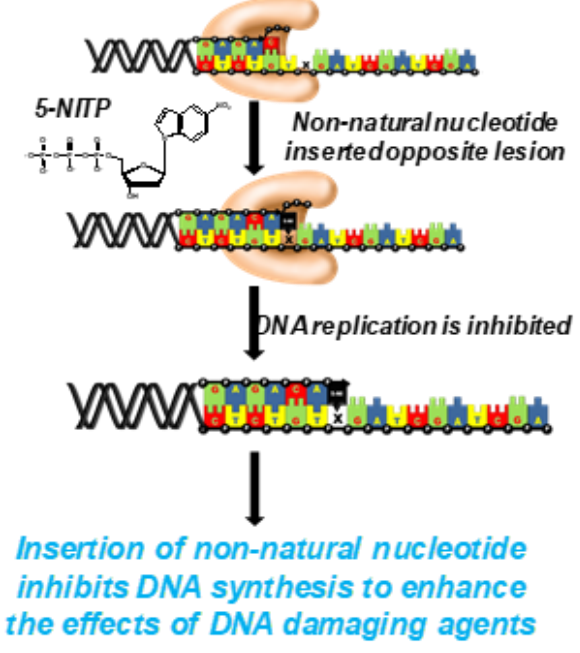

Figure 2 The misreplication of damaged DNA catalyzed by TLS activity can cause mutagenesis in addition to producing drug resistance to anti-cancer agents that produce DNA lesions. One approach to combat these problems is to use artificial nucleotides that can selectively inhibit DNA polymerase involved in replicating DNA lesions generated by therapeutic modalities such as TMZ and ionizing radiation.

This represents a novel approach for several reasons. First, TLS activity can be viewed as the Achilles' heel of GBM cells that are chemoresistant due to defective DNA repair. Secondly, most normal cells are quiescent and possess proficient DNA repair pathways that can effectively correct DNA damage. Since they do not rely on TLS activity for survival unlike their cancerous counterparts, normal cells should be spared from the effects of combining compounds that inhibit TLS activity with low doses of DNA damaging agent. Finally, since TLS activity can be highly pro-mutagenic, it can produce more mutations in a cancer cell to generate more aggressive cancers and/or cause tumor recurrence. In addition, error-prone replication of damaged DNA can transform a normal cell into an oncogenic cell via a phenomenon referred to as "secondary" or "treatment-related" cancers [92]. Thus, inhibiting TLS activity may also be a chemopreventive strategy that prevents these conditions.

To evaluate this approach, the Berdis lab synthesized several artificial nucleotide analogs and characterized their ability to be incorporated opposite an abasic site, the most frequently formed toxic DNA lesion generated by TMZ [93-98]. In vitro studies identified one unique analog, designated 5-NITP, that is efficiently and selectively inserted opposite this DNA lesion (Figure 3) [85]. While 5NITP is efficiently inserted opposite an abasic site, it is refractory to elongation and thus acts as chain terminator against TLS activity. In addition, we demonstrated that 5-NITP is poorly inserted opposite normal DNA (A, C, G, or T). Collectively, these results indicate that the artificial nucleotide retains exquisite selectivity for chain-terminating activity during the replication of an abasic site. 


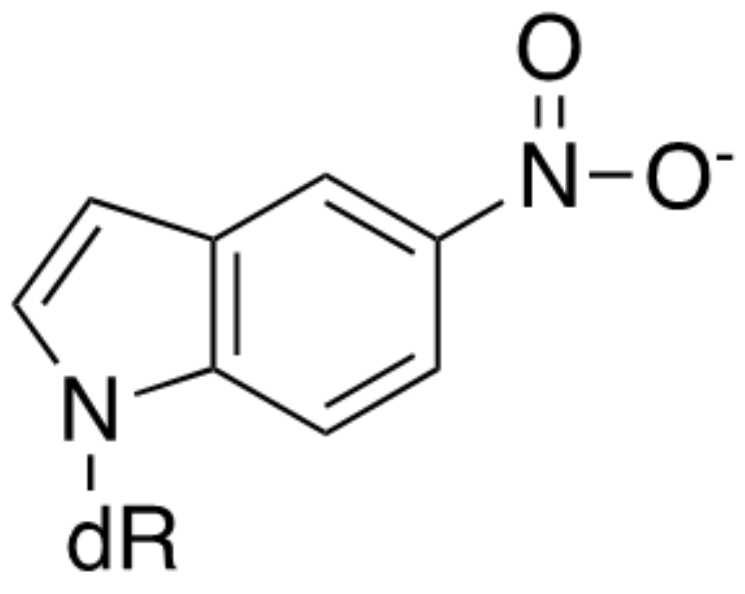

5-NITP

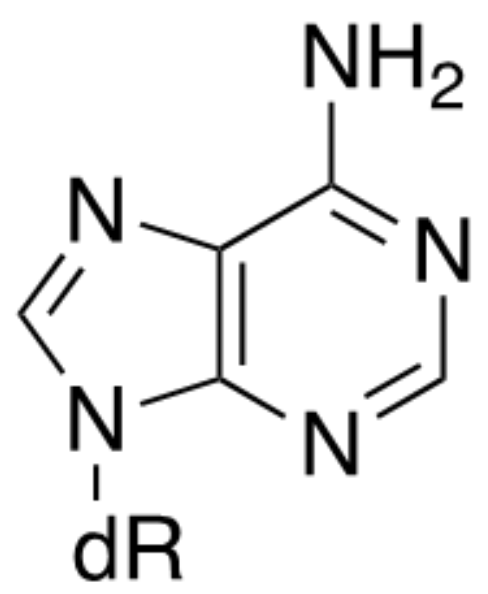

dATP

Figure 3 Structural comparison of the artificial nucleotide analog, 5-nitroindolyl-2'deoxyriboside triphosphate (5-NITP) with the natural nucleotide, dATP.

The ability of several human DNA polymerases to incorporate 5-NITP opposite abasic sites was also examined [98]. These studies included two high-fidelity DNA polymerases (pol $\delta$ and pol $\varepsilon$ ), three specialized human DNA polymerases (pol $\eta$, pol $\iota$, and pol $k$ ), and two DNA polymerases involved in DNA repair ( $\mathrm{pol} \lambda$ and pol $\mu$ ). In vitro analyses demonstrated that both high-fidelity DNA polymerases poorly insert the preferred natural substrate, dATP, opposite an abasic site yet efficiently insert 5-NITP opposite the lesion. Likewise, the specialized DNA polymerases, pol $\eta$ and pol $\mathrm{l}$, insert 5-NITP opposite an abasic site with 100-fold higher efficiencies compared to dATP. However, pol $\kappa$ and the repair DNA polymerases, pol $\lambda$ and pol $\mu$, all replicate an abasic site poorly as none appear to efficiently incorporate dATP and 5-NITP and opposite the lesion.

Cell-based studies using U87 cells as a model, examined if the corresponding nucleoside, 5-NIdR, potentiates the cytotoxic effects of TMZ by inhibiting TLS activity. This human GBM cell line is resistant to $T M Z$ as the $L_{50}$ value for the DNA damaging agent is greater than $100 \mu \mathrm{M}$. Likewise, 5NIdR displays low potency as the $L_{50}$ value is greater than $100 \mu \mathrm{g} / \mathrm{mL}$. In this case, the low potency of 5-NIdR is expected since the corresponding nucleoside triphosphate, 5-NITP, is poorly incorporated opposite undamaged DNA. However, combining 5-NIdR with TMZ significantly increases the cell killing effects of the DNA damaging agent as reflected in 4-fold higher levels of early and late stage apoptosis compared to cells treated individually with TMZ or 5-NIdR. Flow cytometry experiments next evaluated the ability of 5-NIdR alone and in combination with TMZ to affect cell cycle progression. U87 cells treated with $100 \mu \mathrm{g} / \mathrm{mL} 5$-NIdR had no effect on cell cycle progression as compared to control cells treated with vehicle. This result again indicates that the artificial nucleoside generates a minimal cytotoxic effect in the absence of an added DNA damaging agent. However, treatment with $100 \mu \mathrm{M} \mathrm{TMZ}$ produces striking effects on cell cycle progression as there is a large increase in cells at $\mathrm{G}_{2} / \mathrm{M}$ with a concomitant reduction in cells at $\mathrm{G}_{0} / \mathrm{G}_{1}$. These changes occur with minimal effects on cells at $S$-phase or through increases in sub- $G_{1}$ DNA levels. The inability of TMZ to affect S-phase coupled with the accumulation of cells at $\mathrm{G}_{2} / \mathrm{M}$ suggests that 
DNA lesions produced by TMZ do not inhibit chromosomal DNA synthesis and are likely by-passed by specialized DNA polymerases such as pol $\eta$ and/or pol t. Consistent with this hypothesis, combining 5-NIdR with TMZ appears to inhibit TLS activity during S-phase as manifest in a 2-fold increase in cells at S-phase compared to treatment with TMZ or 5-NIdR alone. Furthermore, the increase in sub- $G_{1}$ DNA suggests that cell accumulating at $S$-phase undergo cell death via mitotic catastrophe.

Finally, a heterotopic xenograft mouse model was used to demonstrate that 5-NIdR effectively increases the therapeutic efficacy of TMZ. In these experiments, treatment was initiated with intraperitoneal (IP) injections of TMZ (40 mg/ $\mathrm{kg}$ ) alone or combined with $5-\mathrm{NIdR}(100 \mathrm{mg} / \mathrm{kg})$ for 5 consecutive days once GBM tumors reached a volume of $\sim 500 \mathrm{~mm}^{3}$. TMZ treatment alone had a minimal effect on tumor growth whereas combining 5-NIdR and TMZ produced more significant anti-tumor effects. In particular, the median time for death (MTD) for mice treated with TMZ was 45 days whereas the MTD for mice treated with TMZ and 5-NIdR was greater than 250 days. In fact, the majority of mice ( $>70 \%)$ treated with the drug combination showed complete tumor regression with 30 days post-treatment. Finally, toxicology studies show that repeat dosing with $500 \mathrm{mg} / \mathrm{kg}$ via intravenous injection produces no adverse effects hematological. In addition, no adverse effects on major organs including brain, heart, liver, and kidney were observed in in male and female mice treated with $500 \mathrm{mg} / \mathrm{kg}$.

Other groups have recapitulated this therapeutic strategy by using different approaches to disrupt TLS activity. For example, Ketkar et al. recently synthesized a compound designated IAG-10 ((E)-2-((1-(1-naphthoyl)-5-chloro-1H-indol-3-yl)-methylene)hydrazine-1-carboximidamide hydrochloride) that inhibits human pol $\eta$ activity with a low $\mathrm{IC}_{50}$ value of $\sim 7 \mathrm{M}$ [99] In addition, this compound showed high selectivity against pol $\eta$ as the in vitro activity of other specialized and highfidelity DNA polymerase were unaffected at this concentration of IAG-10. In contrast to the activity of 5-NITP which functions as a selective chain terminating nucleoside analog, IAG-10 inhibits pol $\eta$ activity by disrupting its ability to bind DNA. Using clonogenic cell-based assays, these authors demonstrated that IAG-10 potentiates the cytostatic effects of TMZ in cells that express pol $\eta$. However, IAG-10 did not potentiate TMZ in an isogenic cell line lacking pol $\eta$. Finally, combining IAG10 with TMZ produced an increase in DNA damage as monitored using comet assays to detect SSB formation. It will be of great interest to determine if IAG-10 is effective in an animal model of GBM.

Finally, Wojtaszek et al. reported the identification of a 1,4-dihydroquinolin-4-one derivative designated JH-RE-06 that functions as a small-molecule inhibitor of TLS activity [100]. In this case, JH-RE-06 disrupts TLS by targeting pol $\zeta$ which plays an important role in extending formed mis-pairs rather than catalyzing their formation. Surprisingly, JH-RE-06 appears to bind to a surface of REV1 that normally interacts with the REV7 subunit of pol $\zeta$. As a consequence, the binding of JH-RE-06 to REV1 induces homodimerization which prevents normal REV1-REV7 interactions. This serves to prevent recruitment of pol $\zeta$ to sites of DNA damage to hinder TLS activity. Although not tested against GBM, the authors demonstrated that JH-RE-06 inhibits mutagenic replication catalyzed by TLS activity and increased the cytotoxic effects of cisplatin in human melanoma cells lines. Finally, co-administration of JH-RE-06 with cisplatin lead to significant growth suppression of human melanomas in xenograft mice models. Collectively, these results again reaffirm the validity for using TLS inhibitors as adjunctive agents to improve the therapeutic activity of chemotherapeutic agents that damage DNA. 


\subsection{Conclusions and Future Directions}

While drug resistance to DNA damaging agents is a clinical problem in the treatment of all cancers, it is arguably the most significant issue in the effective treatment of GBM. TMZ is the most widely used anti-cancer agent against GBM, and when combined with radiotherapy, it provides a significant survival benefit for newly diagnosed GBM patients. Unfortunately, TMZ-resistance occurs very rapidly, and this produces a poor prognosis for GBM patients since there are a very limited number of anti-cancer agents that can replace TMZ. Drug resistance is a multi-faceted process and can be caused by increased drug efflux, increased drug metabolism, and increases in levels of glutathione. However, the most common mechanisms associated with TMZ resistance involve alterations in DNA repair pathways including MGMT and MMR (Figure 4).

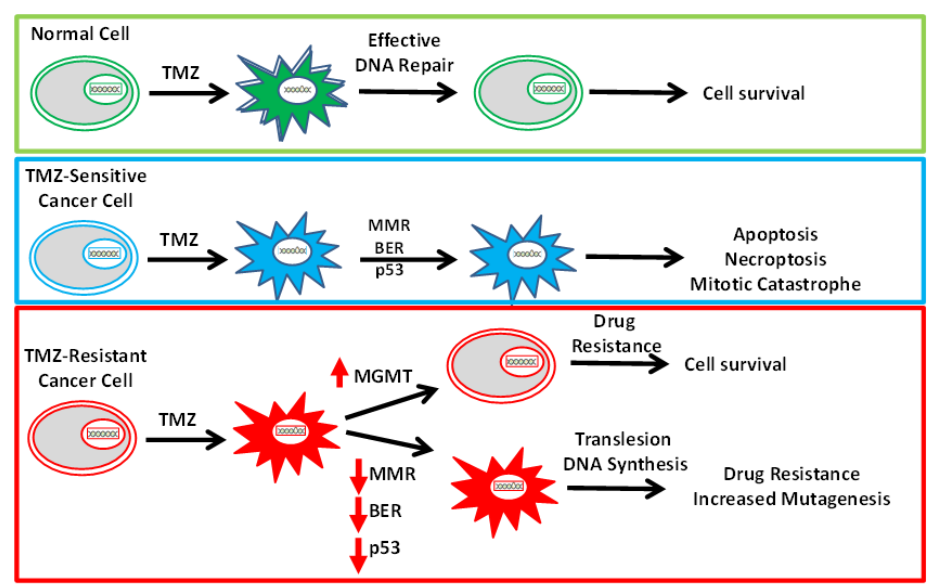

Figure 4 Diagrammatic representation for potential differences in responses and outcomes to DNA damaging agents in a normal cell, a TMZ-sensitive cancer cell, and TMZ-resistant cancer cell. Since most normal cells are quiescent, DNA lesions generated by $T M Z$ can be effectively repaired during the $G_{0}$ phase of the cell cycle. Since DNA replication during S-phase is prohibited, there is a low probability that DNA polymerases will misreplicate the damaged DNA prior to DNA repair. In contrast, both TMZ-sensitive and TMZ-resistant cancer cells have re-entered the cell-cycle to become hyperproliferative. In TMZ-sensitive cancer cells, DNA repair pathways such as MMR and BER are still functional, and their activity ultimately drives these cells toward apoptosis. In TMZ-resistant cancer cells, DNA repair proteins may be overexpressed (MGMT) or either not expressed (MMR) and BER) or mutated (MMR and BER) to generate resistance. In many cases, a lack of functional repair allows DNA lesions to persist, and these damaged formed of DNA are inappropriately replicated to further enhance drug resistance.

In some cases, increased MGMT activity can repair the damage inflicted by TMZ, thus making the drug therapeutically inert. As expected, a decrease in MGMT either through epigenetic mechanisms or through the used of pharmacological inhibitors can, in some cases, reverse TMZ-resistance. This beneficial effect appears related to the activity of the MMR pathway. Unfortunately, a lack of MGMT activity coupled with loss of MMR activity also generates TMZ resistance. These combined defects allow lesions generated by the TMZ to persist and subsequently replicated by several different DNA 
polymerases. Since this activity can be highly pro-mutagenic, it can increase mutational frequencies in a cancer cell to produce more aggressive cancers and/or cause tumor recurrence. As described here, one newly developed approach to combat this problem is to block the ability of DNA polymerases to replicate lesions produced by TMZ. This can be achieved by several different approaches including the use of artificial nucleoside analogs as well as other small molecules that prevent polymerase binding to damaged DNA or that prevent association with other proteins involved in damage recognition.

Despite significant progress in this area, it is important to acknowledge other new approaches being developed to tackle the challenge of TMZ-resistance in GBM. For example, one interesting study by Tso et al. recently used glioblastoma stem cell (GSC) lines expressing MGMT to examine molecular signatures associated with TMZ resistance [101]. Their analyses showed an overall activation of protective stress responses to TMZ treatment which include enzymes associated with biotransformations and detoxification of xenobiotics, blocked endoplasmic reticulum stressmediated apoptosis, epithelial-to-mesenchymal transition (EMT), and inhibited growth/differentiation. One surprising result was the identification of bone morphogenetic protein 7 (BMP7) as the most down-regulated gene in TMZ-resistant GSCs. To verify the importance of this effect, TMZ-resistant GSCs were treated with BMP7 and showed a marked sensitization to the cytotoxic effects of TMZ. In addition, BMP7 treatment caused a loss in self-renewal and migration capacities as well as inducing senescence and downregulation of genes associated with EMT/migration/invasion, stemness, inflammation/immune response, and cell proliferation/tumorigenesis by changing transcriptional profiles. Pre-clinical animal studies showed that BMP7 treatment prolonged survival time of animals intracranially inoculated with GSC compared to untreated mice or mice treated with $\mathrm{TMZ}$ alone. Finally, a more pronounced survival effect was observed by combining BMP7with $T M Z$, thus providing a new potential treatment strategy.

Another interesting approach being developed is the use of Tumor Treating Fields (TTFields). Briefly, this method involves applying alternating electric fields using insulated transducer arrays placed directly in the region surrounding the tumor. A recent study by Giladi et al. showed that applying TTFields against glioma cells lead to a synergistic enhancement in the cell celling effects of radiotherapy [102]. This enhancement appears to be caused by blocking homologous recombination repair of DNA damage caused by radiotherapy. These preclinical results support the application of TTFields therapy immediately after RT as a viable regimen to increased the overall efficacy of radiotherapy in GGM and other cancer patients.

\section{Author Contributions}

Anthony Berdis is the sole author contributing to this work.

\section{Funding}

This work was supported in part through funds made available to the Berdis Glioblastoma Fund.

\section{Competing Interests}

The author has declared that no competing interests exist. 


\section{References}

1. Di Carlo DT, Cagnazzo F, Benedetto N, Morganti R, Perrini P. Multiple high-grade gliomas: Epidemiology, management, and outcome. A systematic review and meta-analysis. Neurosurg Rev. 2019; 42: 263-275.

2. Gallego O. Nonsurgical treatment of recurrent glioblastoma. Curr Oncol. 2015; 22: e273-e281.

3. Stupp R, Pavlidis N, Jelic S. ESMO minimum clinical recommendations for diagnosis, treatment and follow-up of malignant glioma. Ann Oncol. 2005: 16: i64-i65.

4. Cohen $\mathrm{MH}$, Johnson JR, Pazdur R. Food and drug administration drug approval summary: Temozolomide plus radiation therapy for the treatment of newly diagnosed glioblastoma multiforme. Clin Cancer Res. 2005; 11: 6767-6771.

5. Schreck KC, Grossman SA. Role of temozolomide in the treatment of cancers involving the central nervous system. Oncology. 2018; 32: 555-560.

6. Miller KD, Siegel RL, Lin CC, Mariotto AB, Kramer JL, Rowland JH, et al. Cancer treatment and survivorship statistics, 2016. CA Cancer J Clin. 2016; 66: 271-289.

7. Nikolova T, Roos WP, Krämer OH, Strik HM, Kaina B. Chloroethylating nitrosoureas in cancer therapy: DNA damage, repair and cell death signaling. Biochim Biophys Acta Rev Cancer. 2017; 1868: 29-39.

8. Ashby LS, Smith KA, Stea B. Gliadel wafer implantation combined with standard radiotherapy and concurrent followed by adjuvant temozolomide for treatment of newly diagnosed highgrade glioma: A systematic literature review. World J Surg Oncol. 2016; 14: 225.

9. Champeaux C, Weller J. Implantation of carmustine wafers (gliadel) for high-grade glioma treatment. A 9-year nationwide retrospective study. J Neurooncol. 2020; 147: 159-169.

10. Mao Z, Shen K, Zhu L, Xu M, Yu F, Xue D, et al. Comparisons of cardiotoxicity and efficacy of anthracycline-based therapies in breast cancer: A network meta-analysis of randomized clinical trials. Oncol Res Treat. 2019; 42: 405-413.

11. Chamberlain FE, Jones RL, Chawla SP. Aldoxorubicin in soft tissue sarcomas. Future Oncol. 2019; 15: 1429-1435.

12. Groves MD, Portnow J, Boulmay BC, Chawla SP, Dinh H, Chawla S, et al. Phase 2 study of aldoxorubicin in relapsed glioblastoma. J Clin Oncol. 2016; 34: 2027-2027.

13. Carter TC, Medina-Flores R, Lawler BE. Glioblastoma treatment with temozolomide and bevacizumab and overall survival in a rural tertiary healthcare practice. Biomed Res Int. 2018; 31: 6204676.

14. Seystahl K, Hentschel B, Loew S, Gramatzki D, Felsberg J, Herrlinger U, et al. Bevacizumab versus alkylating chemotherapy in recurrent glioblastoma. J Cancer Res Clin Oncol. 2020; 146: 659-670.

15. Fu D, Calvo JA, Samson LD. Balancing repair and tolerance of DNA damage caused by alkylating agents. Nat Rev Cancer. 2012; 12: 104-120.

16. Zhang JH, Malcolm FG, Tracey DB. Temozolomide: Mechanisms of action, repair and resistance. Curr Mol Pharmacol. 2012; 5: 102-114.

17. Sedgwick B, Bates PA, Paik J, Jacobs SC, Lindahl T. Repair of alkylated DNA: Recent advances. DNA Repair (Amst). 2007; 6: 429-442.

18. D'Atri S, Tentori L, Lacal PM, Graziani G, Pagani E, Benincasa E, et al. Involvement of the mismatch repair system in temozolomide-induced apoptosis. Mol Pharmacol. 1998; 54: 334341. 
19. Aasland D, Götzinger L, Hauck L, Berte N, Meyer J, Effenberger M, et al. Temozolomide induces senescence and repression of DNA repair pathways in glioblastoma cells via activation of ATRCHK1, p21, and NF-KB. Cancer Res. 2019; 79: 99-113.

20. Plosky BS, Frank EG, Berry DA, Vennall GP, McDonald JP, Woodgate R. Eukaryotic Y-family polymerases bypass a 3-methyl-2'-deoxyadenosine analog in vitro and methyl methanesulfonate-induced DNA damage in vivo. Nucleic Acids Res. 2008; 36: 2152-2162.

21. Myers K, Gagou ME, Zuazua-Villar P, Rodriguez R, Meuth M. ATR and Chk1 suppress a caspase3-dependent apoptotic response following DNA replication stress. PLoS Genet. 2009; 5: e1000324.

22. Roos WP, Kaina B. DNA damage-induced cell death: From specific DNA lesions to the DNA damage response and apoptosis. Cancer Lett. 2013; 332: 237-248.

23. Quiros S, Roos WP, Kaina B. Processing of $\mathrm{O}^{6}$-methylguanine into DNA double-strand breaks requires two rounds of replication whereas apoptosis is also induced in subsequent cell cycles. Cell Cycle. 2010; 9: 168-178.

24. Loeb LA, Preston BD, Snow ET, Schaaper RM. Apurinic sites as common intermediates in mutagenesis. Basic Life Sci. 1986; 38: 341-347.

25. Loeb LA, Preston BD. Mutagenesis by apurinic/apyrimidinic sites. Annu Rev Genet. 1986; 20 : 201-230.

26. Shrivastav N, Li DY, Essigmann JM. Chemical biology of mutagenesis and DNA repair: Cellular responses to DNA alkylation. Carcinogenesis. 2010; 31: 59-70.

27. Wilson DM, Barsky D. The major human abasic endonuclease: Formation, consequences and repair of abasic lesions in DNA. Mutat Res. 2001; 485: 283-307.

28. Berdis AJ. Dynamics of translesion DNA synthesis catalyzed by the bacteriophage T4 exonuclease-deficient DNA polymerase. Biochemistry. 2001; 40: 7180-7191.

29. Sabouri N, Johansson E. Translesion synthesis of abasic sites by yeast DNA polymerase epsilon. J Biol Chem. 2009; 284: 31555-31563.

30. Baer JC, Freeman AA, Newlands ES, Watson AJ, Rafferty JA, Margison GP. Depletion of O $^{6}$ alkylguanine-DNA alkyltransferase correlates with potentiation of temozolomide and CCNU toxicity in human tumour cells. Br J Cancer. 1993; 67: 1299-1302.

31. Kanzawa T, Bedwell J, Kondo Y, Kondo S, Germano IM. Inhibition of DNA repair for sensitizing resistant glioma cells to temozolomide. J Neurosurg. 2003; 99: 1047-1052.

32. Agnihotri S, Gajadhar AS, Ternamian C. Alkylpurine-DNA-N-glycosylase confers resistance to temozolomide in xenograft models of glioblastoma multiforme and is associated with poor survival in patients. J Clin Invest. 2012; 122: 253-266.

33. Pegg $A E$, Fang $Q$, Loktionova NA. Human variants of $\mathrm{O}^{6}$-alkylguanine-DNA alkyltransferase. DNA Repair (Amst). 2007; 6: 1071-1078.

34. Yu W, Zhang LL, Wei QC, Shao AW. O6-Methylguanine-DNA methyltransferase (MGMT): Challenges and new opportunities in glioma chemotherapy. Front Oncol. 2020; 9: 1547.

35. Li GM. DNA mismatch repair and cancer. Front Biosci. 2003; 8: d997-d1017.

36. Li ZD, Pearlman AH, Hsieh P. DNA mismatch repair and the DNA damage response. DNA Repair (Amst). 2016; 38: 94-101.

37. Zharkov DO. Base excision DNA repair. Cell Mol Life Sci. 2008; 65: 1544-1565.

38. Lee TH, Kang TH. DNA oxidation and excision repair pathways. Int J Mol Sci. 2019; 20: 6092. 
39. Hazra TK, Roy R, Biswas T, Grabowski DT, Pegg AE, Mitra S. Specific recognition of $\mathrm{O}^{6}$ methylguanine in DNA by active site mutants of human $\mathrm{O}^{6}$-methylguanine-DNA methyltransferase. Biochemistry. 1997; 36: 5769-5776.

40. Gouws C, Pretorius PJ. $\mathrm{O}^{6}$-methylguanine-DNA methyltransferase (MGMT): Can function explain a suicidal mechanism? Med Hypotheses. 2011; 77: 857-860.

41. Dolan ME, Pegg AE, Dumenco LL, Moschel RC, Gerson SL. Comparison of the inactivation of mammalian and bacterial $\mathrm{O}^{6}$-alkylguanine-DNA alkyltransferases by $\mathrm{O}^{6}$-benzylguanine and $\mathrm{O}^{6}$ methylguanine. Carcinogenesis. 1991; 12: 2305-2309.

42. Schold SC, Kokkinakis DM, Rudy JL, Moschel RC, Pegg AE. Treatment of human brain tumor xenografts with O6-benzyl-2'-deoxyguanosine and BCNU. Cancer Res. 1996; 56: 2076-2081.

43. Vlachostergios PJ, Hatzidaki E, Papandreou CN. MGMT repletion after treatment of glioblastoma cells with temozolomide and $\mathrm{O}^{6}$-benzylguanine implicates NF KB and mutant p53. Neurol Res. 2013; 35: 879-882.

44. Gusyatiner O, Hegi ME. Glioma epigenetics: From subclassification to novel treatment options. Semin Cancer Biol. 2018; 51: 50-58.

45. van Nifterik KA, van den Berg J, van der Meide WF, Ameziane N, Wedekind LE, Steenbergen RD, et al. Absence of the MGMT protein as well as methylation of the MGMT promoter predict the sensitivity for temozolomide. Br J Cancer. 2010; 103: 29-35.

46. Ma JG, Murphy M, O'Dwyer PJ, Berman E, Reed K, Gallo JM. Biochemical changes associated with a multidrug-resistant phenotype of a human glioma cell line with temozolomide-acquired resistance. Biochem Pharmacol. 2002; 63: 1219-1228.

47. Kitange GJ, Carlson BL, Schroeder MA. Induction of MGMT expression is associated with temozolomide resistance in glioblastoma xenografts. Neuro Oncol. 2009; 11: 281-291.

48. Hegi ME, Liu L, Herman JG, Stupp R, Wick W, Weller M, et al. Correlation of $\mathrm{O}^{6}$-methylguanine methyltransferase (MGMT) promoter methylation with clinical outcomes in glioblastoma and clinical strategies to modulate MGMT activity. J Clin Oncol. 2008; 26: 4189-4199.

49. Sasmita AO, Wong YP, Ling AP. Biomarkers and therapeutic advances in glioblastoma multiforme. Asia Pac J Clin Oncol. 2018; 14: 40-51.

50. Rao AM, Quddusi A, Shamim MS. The significance of MGMT methylation in glioblastoma multiforme prognosis. J Pak Med Assoc. 2018; 68: 1137-1139.

51. Kunkel TA, Erie DA. DNA mismatch repair. Annu Rev Biochem. 2005; 74: 681-710.

52. Lenhart JS, Pillon MC, Guarné A, Biteen JS, Simmons LA. Mismatch repair in Gram-positive bacteria. Res Microbiol. 2016; 167: 4-12.

53. Schofield MJ, Hsieh P. DNA mismatch repair: Molecular mechanisms and biological function. Annu Rev Microbiol. 2003; 57: 579-608.

54. Guarné A, Charbonnier JB. Insights from a decade of biophysical studies on MutL: Roles in strand discrimination and mismatch removal. Prog Biophys Mol Biol. 2015; 117: 149-156.

55. Masih PJ, Kunnev D, Melendy T. Mismatch repair proteins are recruited to replicating DNA through interaction with proliferating cell nuclear antigen (PCNA). Nucleic Acids Res. 2008; 36: 67-75.

56. Surtees JA, Alani E. Replication factors license exonuclease I in mismatch repair. Mol Cell. 2004; 15: 164-166.

57. Lancey C, Tehseen M, Raducanu VS, Rashid F, Merino N, Ragan TJ, et al. Structure of the processive human Pol $\delta$ holoenzyme. Nat Commun. 2020; 11: 1109. 
58. Bowen N, Kolodner RD. Reconstitution of Saccharomyces cerevisiae DNA polymerase $\varepsilon-$ dependent mismatch repair with purified proteins. Proc Natl Acad Sci USA. 2017; 114: 36073612.

59. Xavier A, Olsen MF, Lavik LA, Johansen J, Singh AK, Sjursen W, et al. Comprehensive mismatch repair gene panel identifies variants in patients with Lynch-like syndrome. Mol Genet Genomic Med. 2019; 7: e850.

60. Robertson $A B$, Klunglan $A$, Rognes $T$, Leiros I. DNA repair in mammalian cells: Base excision repair: The long and short of it. Cell Mol Life Sci. 2009; 66: 981-993.

61. Lindahl T. Suppression of spontaneous mutagenesis in human cells by DNA base excision-repair. Mutat Res. 2000; 462: 129-135.

62. Abbotts R, Madhusudan S. Human AP endonuclease 1 (APE1): From mechanistic insights to druggable target in cancer. Cancer Treat Rev. 2010; 36: 425-435.

63. Abe H, Natsumeda M, Okada M, Watanabe J, Tsukamoto Y, Kanemaru Y, et al. MGMT expression contributes to temozolomide resistance in H3K27M-mutant diffuse midline gliomas. Front Oncol. 2020; 9: 1568.

64. Perazzoli G, Prados J, Ortiz R, Caba O, Cabeza L, Berdasco M, et al. Temozolomide resistance in glioblastoma cell lines: Implication of MGMT, MMR, P-glycoprotein and CD133 expression. PLoS One. 2015; 10: e0140131.

65. Johnson BE, Mazor T, Hong C, Barnes M, Aihara K, McLean CY, et al. Mutational analysis reveals the origin and therapy-driven evolution of recurrent glioma. Science. 2014; 343: 189-193.

66. Pham P, Rangarajan S, Woodgate R, Goodman MF. Roles of DNA polymerases V and II in SOSinduced error-prone and error-free repair in Escherichia coli. Proc Natl Acad Sci USA. 2001; 98 : 8350-8354.

67. Sutton MD, Walker GC. Managing DNA polymerases: coordinating DNA replication, DNA repair, and DNA recombination. Proc Natl Acad Sci USA. 2001; 98: 8342-8349.

68. Guo J, Zhou GL, Zhang WF, Song YL, Bian Z. A novel POLH mutation causes XP-V disease and XP$\checkmark$ tumor proneness may involve imbalance of numerous DNA polymerases. Oncol Lett. 2013; 6 : 1583-1590.

69. Stary A, Kannouche $P$, Lehmann AR, Sarasin A. Role of DNA polymerase eta in the UV mutation spectrum in human cells. J Biol Chem. 2013; 278: 18767-18775.

70. Teng KY, Qiu MZ, Li ZH, Luo HY, Zeng ZL, Luo RZ, et al. DNA polymerase $\eta$ protein expression predicts treatment response and survival of metastatic gastric adenocarcinoma patients treated with oxaliplatin-based chemotherapy. J Transl Med. 2010; 8: 126.

71. Matsuda T, Bebenek K, Masutani C, Hanaoka F, Kunkel TA. Low fidelity DNA synthesis by human DNA polymerase-eta. Nature. 2000; 404: 1011-1013.

72. Kokoska RJ, McCulloch SD, Kunkel TA. The efficiency and specificity of apurinic/apyrimidinic site bypass by human DNA polymerase eta and sulfolobus solfataricus Dpo4. J Biol Chem. 2003; 278: 50537-50545.

73. Haracska L, Prakash S, Prakash L. Replication past $\mathrm{O}^{6}$-methylguanine by yeast and human DNA polymerase $\eta$. Mol Cell Biol. 2000; 20: 8001-8007.

74. Yoon JH, Prakash L, Prakash S. Highly error-free role of DNA polymerase eta in the replicative bypass of UV-induced pyrimidine dimers in mouse and human cells. Proc Natl Acad Sci USA. 2009; 106: 18219-18224. 
75. Reha-Krantz LJ, Nonay RL, Day RS, Wilson SH. Replication of $\mathrm{O}^{6}$-methylguanine-containing DNA by repair and replicative DNA polymerases. J Biol Chem. 1996; 271: 20088-20095.

76. Chavarria D, Ramos-Serrano A, Hirao I, Berdis AJ. Exploring the roles of nucleobase desolvation and shape complementarity during the misreplication of $\mathrm{O}^{6}$-methylguanine. J Mol Biol. 2011; 412: 325-339.

77. Jałoszyński $\mathrm{P}$, Ohashi E, Ohmori H, Nishimura S. Error-prone and inefficient replication across 8hydroxyguanine (8-oxoguanine) in human and mouse ras gene fragments by DNA polymerase. Genes Cells. 2005; 10: 543-550.

78. Nick McElhinny SA, Havener JM, Garcia-Diaz M, Juárez R, Bebenek K, Kee BL, et al. A gradient of template dependence defines distinct biological roles for family $X$ polymerases in nonhomologous end joining. Mol Cell. 2005; 19: 357-366.

79. Bertocci B, De Smet A, Weill JC, Reynaud CA. Nonoverlapping functions of DNA polymerases mu, lambda, and terminal deoxynucleotidyltransferase during immunoglobulin $V(D) J$ recombination in vivo. Immunity. 2006; 25: 31-41.

80. Sugiyama $T$, Chen YZ. Biochemical reconstitution of UV-induced mutational processes. Nucleic Acids Res. 2019; 47: 6769-6782.

81. Zhu XZ, Zou ST, Zhou JD, Zhu HS, Zhang SY, Shang ZF, et al. REV3L, the catalytic subunit of DNA polymerase $\zeta$, is involved in the progression and chemoresistance of esophageal squamous cell carcinoma. Oncol Rep. 2016; 35: 1664-1670.

82. Lin XJ, Trang J, Okuda T, Howell SB. DNA polymerase zeta accounts for the reduced cytotoxicity and enhanced mutagenicity of cisplatin in human colon carcinoma cells that have lost DNA mismatch repair. Clin Cancer Res. 2006; 12: 563-568.

83. Wang WJ, Sheng WJ, Yu CX, Cao JP, Zhou JD, Wu JC, et al. REV3L modulates cisplatin sensitivity of non-small cell lung cancer H1299 cells. Oncol Rep. 2015; 34: 1460-1468.

84. Vassel FM, Bian K, Walker GC, Hemann MT. Rev7 loss alters cisplatin response and increases drug efficacy in chemotherapy-resistant lung cancer. Proc Natl Acad Sci USA. 2020; 117: 2892228924.

85. Yasui M, Suzuki N, Laxmi YR, and Shibutani S. Translesion synthesis past tamoxifen-derived DNA adducts by human DNA polymerases $\eta$ and $k$. Biochemistry. 2006; 45: 12167-12174.

86. Zhou WD, Chen YW, Liu XY, Chu PG, Loria S, Wang YF, et al. Expression of DNA translesion synthesis polymerase $\eta$ in head and neck squamous cell cancer predicts resistance to gemcitabine and cisplatin-based chemotherapy. PLoS One. 2013; 8: e83978.

87. Wang HB, Wu WT, Wang HW, Wang S, Chen YY, Zhang XT, et al. Analysis of specialized DNA polymerases expression in human gliomas: Association with prognostic significance. Neuro Oncol. 2010; 12: 679-686.

88. Peng $\mathrm{CH}$, Chen ZX, Wang S, Wang HW, Qiu WJ, Zhao L, et al. The error-prone DNA polymerase $\mathrm{K}$ promotes temozolomide resistance in glioblastoma through Rad17-dependent activation of ATR-Chk1 signaling. Cancer Res. 2015; 76: 2340-2353.

89. Bostian AC, Maddukuri L, Reed MR, Savenka T, Hartman JH, Davis L, et al. Kynurenine signaling increases DNA polymerase kappa expression and promotes genomic instability in glioblastoma cells. Chem Res Toxicol. 2016; 29: 101-108.

90. Campbell BB, Light N, Fabrizio D, Zatzman M, Fuligni F, de Borja R, et al. Comprehensive analysis of hypermutation in human cancer. Cell. 2017; 171: 1042-1056. 
91. Choi JS, Kim S, Motea E, Berdis A. Inhibiting translesion DNA synthesis as an approach to combat drug resistance to DNA damaging agents. Oncotarget. 2017; 8: 40804-40816.

92. Demoor-Goldschmidt C, de Vathaire F. Review of risk factors of secondary cancers among cancer survivors. Br J Radiol. 2019; 92: 20180390.

93. Zhang XM, Lee I, Berdis AJ. Evaluating the contributions of desolvation and base-stacking during translesion DNA synthesis. Org Biomol Chem. 2004; 2: 1703-1711.

94. Zhang XM, Lee I, Berdis AJ. The use of nonnatural nucleotides to probe the contributions of shape complementarity and pi-electron surface area during DNA polymerization. Biochemistry. 2005; 44: 13101-13110.

95. Zhang XM, Lee I, Zhou X, Berdis AJ. Hydrophobicity, shape, and pi-electron contributions during translesion DNA synthesis. J Am Chem Soc. 2006; 128: 143-149.

96. Vineyard D, Zhang XM, Donnelly A, Lee I, Berdis AJ. Optimization of non-natural nucleotides for selective incorporation opposite damaged DNA. Org Biomol Chem. 2007; 5: 3623-3630.

97. Reineks EZ, Berdis AJ. Evaluating the contribution of base stacking during translesion DNA replication. Biochemistry. 2004; 43: 393-404.

98. Choi JS, Kim CS, Berdis A. Inhibition of translesion DNA synthesis as a novel therapeutic strategy to treat brain cancer. Cancer Res. 2018; 78: 1083-1096.

99. Ketkar A, Maddukuri L, Penthala NR, Reed MR, Zafar MK, Crooks PA, et al. Inhibition of human DNA polymerases eta and kappa by indole-derived molecules occurs through distinct mechanisms. ACS Chem Biol. 2019; 14: 1337-1351.

100.Wojtaszek JL, Chatterjee N, Najeeb J, Ramos A, Lee M, Bian K, et al. A small molecule targeting mutagenic translesion synthesis improves chemotherapy. Cell. 2019; 178: 152-159.

101.Tso JL, Yang S, Menjivar JC, Yamada K, Zhang Y, Hong I, et al. Bone morphogenetic protein 7 sensitizes $\mathrm{O}^{6}$-methylguanine methyltransferase expressing-glioblastoma stem cells to clinically relevant dose of temozolomide. Mol Cancer. 2015; 14: 1-17.

102.Giladi M, Munster M, Schneiderman RS, Voloshin T, Porat $Y$, Blat R, et al. Tumor treating fields (TTFields) delay DNA damage repair following radiation treatment of glioma cells. Radiat Oncol. 2017; 12: 1-13.

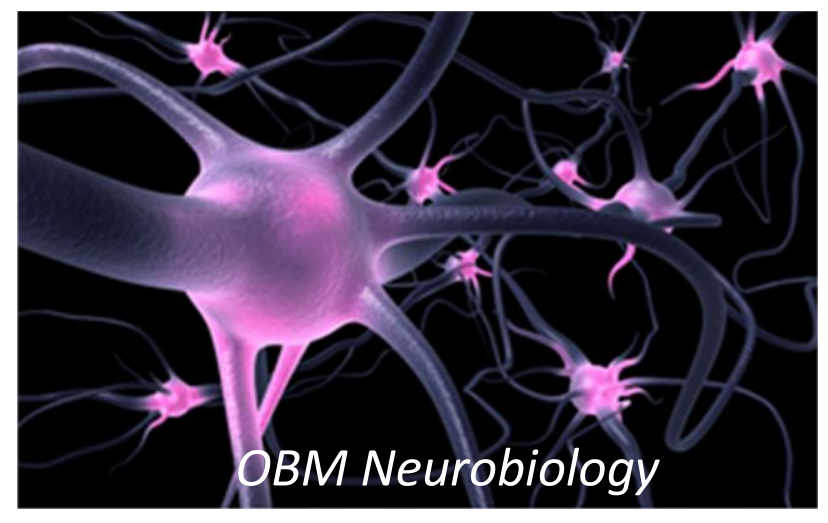

Enjoy OBM Neurobiology by:

1. Submitting a manuscript

2. Joining volunteer reviewer bank

3. Joining Editorial Board

4. Guest editing a special issue

For more details, please visit:

http://www.lidsen.com/journals/neurobiology 